\title{
Giovanni Paoletti, Benjamin Constant et les Anciens: politique, religion, histoire
}

\section{François Rosset}

\section{(2) OpenEdition}

\section{Journals}

Édition électronique

URL : http://journals.openedition.org/studifrancesi/8954

DOI : 10.4000/studifrancesi.8954

ISSN : 2427-5856

Éditeur

Rosenberg \& Sellier

\section{Édition imprimée}

Date de publication : 1 octobre 2008

Pagination : 467-468

ISSN : 0039-2944

\section{Référence électronique}

François Rosset, "Giovanni Paoletti, Benjamin Constant et les Anciens: politique, religion, histoire », Studi Francesi [En ligne], 155 (LII | II) | 2008, mis en ligne le 30 novembre 2015, consulté le 10 janvier 2021. URL : http://journals.openedition.org/studifrancesi/8954; DOI : https://doi.org/10.4000/studifrancesi. 8954

Ce document a été généré automatiquement le 10 janvier 2021.

\section{(c) $($ ) $\odot$ (8)}

Studi Francesi è distribuita con Licenza Creative Commons Attribuzione - Non commerciale - Non opere derivate 4.0 Internazionale. 


\title{
Giovanni Paoletti, Benjamin Constant et les Anciens: politique, religion, histoire
}

\author{
François Rosset
}

\section{RÉFÉRENCE}

GIOVANNI PAOLETTI, Benjamin Constant et les Anciens: politique, religion, histoire, traduit de l'italien par Marie-France MERGER, Paris, Honoré Champion, 2006 («Travaux et recherches de l'Institut Benjamin Constant»), pp. 468.

1 Dès sa parution originale en italien, en 2001, cet ouvrage fut remarqué par les spécialistes, au point qu'il parut urgent d'en proposer une diffusion plus large par le biais d'une édition française. Abordant la pensée et l'œuvre de Constant dans l'optique apparemment restreinte de la question des Anciens, trop souvent examinée à la seule lumière du fameux discours De la Liberté des anciens comparée à celle des modernes, G. Paoletti met au jour la permanence et la richesse de cette problématique tout au long du parcours intellectuel de Constant et dans la diversité de son œuvre politique, littéraire et historique. Finalement, cette étude spécialisée prend l'ampleur d'une monographie très riche et stimulante; c'est l'un des ouvrages les plus importants consacrés à l'auteur d'Adolphe ces dernières décennies.

L'ouvrage présente une cohérence de pensée remarquable. Deux grandes directions s'y articulent: celle qui consiste à considérer l'histoire même des anciens et celle qui propose une interrogation sur l'investissement de cette histoire dans la pensée politique. C'est une distinction qui touche aussi bien les objets de l'étude que la démarche de l'étude elle-même, mais une distinction non schématique qui conduit le lecteur avec souplesse dans une démonstration dominée par le souci de la nuance. Le livre est ainsi divisé en deux grandes parties «Imiter les Anciens?» et «Étudier les Anciens», chacune d'elles comportant à son tour deux volets pour une somme de quatre 
chapitres progressifs («Le vertige du passé», «Illusions et politique», «La modernité vue d'ailleurs», "L'"heureuse exception"»). La structure le montre ainsi d'emblée: nous ne sommes pas dans un régime de pensée radicalement binaire, mais dans une marche qui avance par le balancement même entre deux modalités d'approche complémentaires.

Le premier chapitre offre une mise en contexte des conceptions de Constant. Dans une présentation synthétique remarquable de clarté, Paoletti expose les différentes tendances du débat amorcé pendant et juste après la Révolution sur le classicisme des Jacobins. Deux éléments sont pris en compte: l'évaluation de la justesse historique de leurs références antiques et celle de la pertinence politique de leur recours à celles-ci. La combinaison de ces deux ordres d'éléments permet de distinguer quatre positions: celle attribuée aux Jacobins eux-mêmes, sont sûrs de l'une et de l'autre; celle de leurs adversaires radicaux qui récusent l'une et l'autre (par exemple, Volney); celle de ceux qui leur concèdent une certaine justesse historique, mais pas de pertinence politique (telle Mme de Staël); ceux qui, à l'inverse, dénient la justesse historique tout en reconnaissant une certaine pertinence politique (ainsi de Chateaubriand et Constant). Le propos n'est évidemment pas aussi schématique; il distingue clairement, tout en apportant beaucoup de nuances. Des propositions assez originales apparaissent même dans ces propos "topographiques», telle la lecture très attentive du discours des Jacobins qui n'apparait pas aussi univoque qu'on l'attendait, la présentation du point de vue de Chateaubriand dans l'Essai sur les Révolutions et surtout les développements conclusifs qui présentent un parallèle entre les idées de Constant et celles de Karl Marx.

4 Le deuxième chapitre est plus "conceptuel» et offre au philosophe toute la latitude nécessaire pour développer ses réflexions autour de la notion d'illusion. Au point de départ de cette analyse, il y a le rappel des conditions de pensée nouvelles instaurées par la Révolution par rapport à l'examen des erreurs et des préjugés propres à l'héritage des Lumières. «Les égarements d'hommes bien intentionnés et même éclairés, le rôle actif joué par le peuple, la suppression de la liberté au nom de la liberté elle-même, la tentation de se soumettre à un nouveau despotisme» poussent à réexaminer l'étude des rapports entre le vrai et le faux, entre la réalité et les apparences. L'exemple des Anciens permet alors à Constant de mettre en évidence la nouveauté que l'histoire la plus récente a imposée pour considérer les mécanismes d'aliénation de la liberté. En recourant à la notion d'illusion, il montre notamment comment la tyrannie s'impose sans avoir été programmée (au contraire des modèles anciens du despotisme) et surtout pourquoi le rapport entre gouvernants et gouvernés n'est pas seulement l'affaire d'une application de principes, de stratégies et de lois. Car est en jeu également la dimension de la subjectivité humaine qu'il faut mettre au jour pour essayer de comprendre pourquoi les sujets peuvent se soumettre à la tyrannie, comment on peut croire être libre sans l'être réellement, voire même encourager la tyrannie tout en étant convaincu de défendre la liberté. La démonstration passe par l'évocation des philosophes qui inspirent Constant (en particulier Rousseau), mais elle intègre aussi des pages très fines où sont décrits les instruments langagiers qui soutiennent l'illusion. Ainsi, le chapitre s'achève sur une analyse subtile de deux passages d'Adolphe où l'évocation du paysage permet de mettre en relief tout le registre métaphorique du brouillard, de l'indéterminé et de l'indéfini auquel l'auteur a si souvent recours dans d'autres textes à vocation toute différente.

5 Dans le troisième chapitre, l'enquête aborde à bras le corps la question de la connaissance que Constant avait de la culture antique et de l'exploitation qu'il en a 
faite dans ses écrits politiques. Une fois encore, on peut distinguer deux ordres d'observations: il y a le Constant mû par sa fibre d'historien, qui examine le monde antique pour lui-même, et celui qui, penché sur l'observation et la critique du présent, puise des illustrations, des exemples et des contre-exemples dans le grand réservoir du monde ancien. En mettant au jour de façon très précise l'oscillation entre ces deux manières de voir, Paoletti montre parfaitement comment Constant se donne les moyens d'analyser le monde contemporain à partir d'une certaine distance; c'est ce qu'il appelle «la modernité vue d'ailleurs».

6 Le quatrième chapitre est presque entièrement consacré à la réflexion de Constant sur la religion et à ses immenses travaux d'historien des formes religieuses. Paoletti rappelle tout d'abord les étapes du long parcours de Constant élaborant son grand ouvrage pendant des décennies en changeant plusieurs fois de projet. Est mis en évidence, comme l'étape essentielle de ce parcours, le moment où le penseur s'est libéré de la distinction courante entre polythéisme et théisme pour fonder sa réflexion sur l'opposition entre religions sacerdotales et religions libres ou indépendantes du sacerdoce. C'est là bien sûr que se noue l'articulation, autour de la notion de liberté, entre la pensée politique et la vision religieuse de Constant. Lu dans cette perspective, le traité De la Religion apparaît comme «un ouvrage consacré au rapport conflictuel entre l'expérience de la liberté et l'acquiescement à la servitude». On appréciera tout particulièrement, dans ce chapitre, les pages consacrées au problème du mal traité à la lumière de la figure emblématique de Philoctète; la réflexion de Constant qui s'affranchit de la vision voltairienne du mal comme culpabilité se tourne vers l'univers de la tragédie où le mal est considéré comme destinée. On le voit, il ne saurait y avoir, chez Constant, en matière de religion comme en politique, de théorie ou d'histoire sans anthropologie.

7 Cette dernière observation nous permet aussi de conclure en soulignant l'intérêt et la richesse de cette étude fondée sur un sujet en apparence restreint, mais qui se développe en embrassant un champ toujours plus vaste pour livrer finalement un examen global et très profond de la pensée et des écrits de Benjamin Constant. 